I have already mentioned, I have known Dr. Adler for many years and I have followed his progress with the greatest interest and greatest joy.

Chairman, Professor Emil Adler. We have a very tight schedule, but before we start with the lectures I have a few remarks to make. First of all, I have to thank Sir Ludwig for his very kind words in introducing me. I don't know whether I merit such praise. I am not going to repeat again what we feel with regard to Dr. Ludwig Guttmann and what I have felt for many, many years.

Secondly, I have been asked to say a few words on behalf of the Medical School and the Medical Faculty. Unfortunately, a very short time ago, our Dean, Professor Halperin, who would have been greatly interested in this Meeting, passed away. He was one of our best friends and one of the most important personalities in Neurology in this country-and not only in this country-and we are at present orphans as far as the Deanship goes. I have been asked on behalf of the Medical School to greet this Meeting and to wish it complete success.

Our first subject is Deformity of the Spine and we have five speakers on this subject in the morning session. The first is Mr. McSweeney from Great Britain, a well-known orthopaedic surgeon in Great Britain. I don't think he requires very much introduction, as most of the people here know him very well, and I should like him to take the floor.

\title{
SPINAL DEFORMITY AFTER SPINAL CORD INJURY
}

\author{
By T. MCSweEneY, M.Ch.(N.U.I.), M.Ch.(Orth.), F.R.C.S.(England) \\ The Robert Fones and Agnes Hunt Orthopaedic Hospital, Oswestry
}

\section{INTRODUCTION}

THE return of tone in the spinal muscles after spinal cord injury is such that gross deformity of the spinal column in properly treated adult patients is unusual. In children there may be little or no alteration in spinal alignment in the early months after injury; as the child grows minor asymmetry of muscle balance may lead to progressive deformity. The principle of homeostasis, whereby the damaged spinal cord maintains near normal spinal alignment, is conditioned by the recovery of tone in the spinal muscles, which in turn depends on the proper early and continued management of the patient. The time is long since past when complete lesions were associated with the notion of paraplegia-in-flexion; its avoidance depends on the proper posturing of the patient, early passive exercises, the treatment of sepsis, and many other details of management which require no further emphasis here. As Guttmann (1952) pointed out in his Wartenberg lecture'The conception that paraplegia-in-flexion is pathognomonic of complete transection, while paraplegia-in-extension is pathognomonic of incomplete lesions of the spinal cord, is no longer valid in its dogmatic form'. 


\section{FACTORS CONCERNED IN SPINAL ALIGNMENT}

The shape of the vertebral bodies, the arrangement of the intervertebral discs, the disposition of the ligaments and joints, and the pressure exerted by the ribs are the main factors that preserve the curvatures of the normal adult spine, subject always to the controlling influence of muscle action. Muscle imbalance has long been recognised as a potent factor in bone and joint deformity. Perverted muscle action can produce functional as well as later structural changes in the spine.

Roaf (I966) has stressed that the normal action of the superficial or longitudinal fibres of the erector spinae group is to extend the spine. Normally this action is balanced by a number of factors, which include gravity, and the action of the opposing anterior cervical and anterior abdominal muscles. Roaf has further emphasised that when a rotational element is present the longitudinal fibres tend to increase the deformity. Contraction of the deep or oblique fibres of the erector spinae rotates the upper vertebra to the opposite side. Asymmetrical action of the deep fibres may well be one of the important factors in initiating a rotational deformity.

With advancing years the spinal column loses much of its plasticity and flexibility, so that disorders of the neuromuscular mechanisms produce less deformity in older spines. In childhood and adolescence the column is less rigid and more sensitive to minor alterations in muscle balance.

In pathological conditions of the cord following injury (or disease) the effect on muscle balance is one of great complexity. When the cord lesion is complete, the stretch reflexes are usually sufficient to restore a state of almost normal muscle 'tone' distal to the site of injury. (This applies in general to lesions above the conus and unassociated with massive necrosis of the cord.) These simple responses are modified by the more complex polysynaptic intersegmental reflexes as cord autonomy develops. The intrinsic spinal reflexes may occur at any level of the neuroaxis which has a primary afferent from the periphery and a primary efferent to an effector organ (muscle). The discharge through the efferent fibres from the anterior horn cells is modified by a multiplicity of neural influences from extroceptive, proprioceptive and introceptive receptors. Furthermore, any discharge of impulses along the final common pathway is influenced by the central excitatory and central inhibitory state of the spinal cord. Intrinsic mechanisms become established once the stage of spinal shock has passed off but are influenced by a number of factors. These include prolonged recumbency, sepsis, urinary infections, deformities elsewhere, and emotion. Intrinsic reflexes are favoured by remedial exercises for the unparalysed muscles and by passive mobilisation at all stages.

Synaptic transmission and the passage of impulses across the myoneural junction are mediated by chemical processes.

Despite disappointments to date, there is little doubt that with a greater understanding of these complex mechanisms, their pharmacological manipulation is not too remote.

Complete lesions below the conus are associated with a flaccid paralysis of lower motor neurone type, but this does not preclude the development of secondary deformities. When the lesion is incomplete the lower limb deformities are likely to be more progressive.

There is a small group of patients in whom tonic reflex activity fails to return, so that the spinal muscles remain flaccid and many muscle segments may develop atrophy after the manner of a lower motor neurone lesion. In these patients it is 
probable either that massive necrosis of the anterior horn cells has resulted from the injury, or massive longitudinal vascular damage with cord infarction has occurred around the level of the lesion (figs I and 2).

Before considering the factors influencing spinal deformity resulting from injury to the cord it is interesting to reflect on how little is known of the mechanisms concerned in the production of the spinal deformity encountered in a wide variety of primary neurological disorders, from the congenital myopathies to postencephalitic Parkinsonism. Neurologists have long been aware of the scoliosis

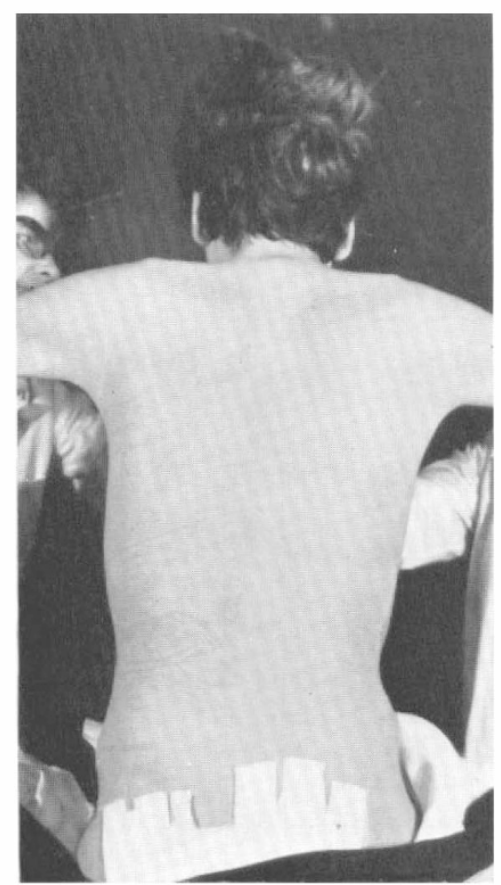

FIG. I

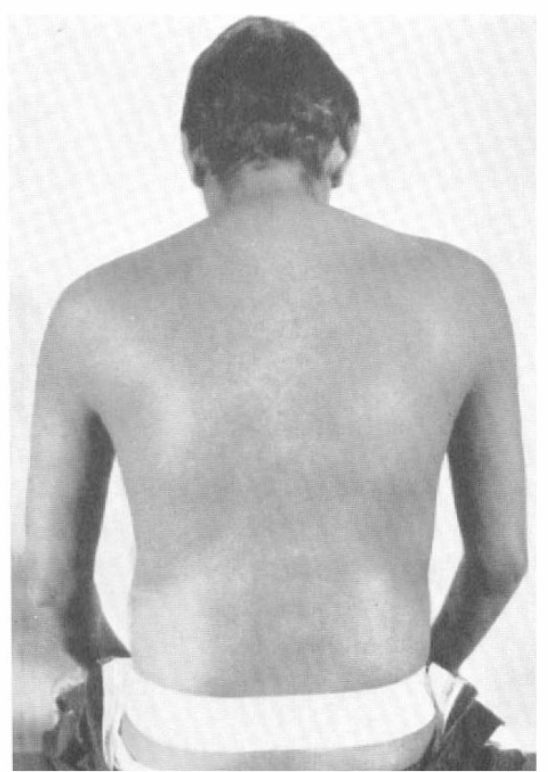

FIG. 2

Fig. I.-Failure to recover muscle tone two years after severe compression fractures of C.V. 5 and 6. Longitudinal vascular damage was suspected.

Fig. 2.-Good recovery of muscle tone six months after complete lesion at C6. (Contrast with Figure I.)

which often accompanies the muscular dystrophies, infantile spinal progressive muscular atrophy (Werdnig-Hoffman disease), syringomyelia, the spino-cerebellar degenerations (Friedreich's ataxia), neurofibromatosis (Von Recklinghausen's disease), and spinal dysraphism. In some of these the deformity may be due to a primary muscle disorder, but in many there is a derangement of postural activity because of interference with proprioceptive afferents to the cord, and in others there is a derangement of the supraspinal centres involved in postural activity.

Quite apart from muscle weakness, fibrotic shortening or contracture of muscles, such as occurs in arthrogryposis multiplex congenita and polymyositis, is sometimes encountered as a cause of spinal deformity. This is mentioned 
because of the analogy with spastic contractures in spinal injury patients, and with the fibrosis of muscle which may follow extensive operative exposure of the spinal column. Orthopaedic surgeons are only too well aware of the enigma presented by so-called idiopathic scoliosis. When anterior poliomyelitis was a common disease the picture presented by paralytic curvature was a comparatively simple mechanical one. We were dealing with a lower motor neurone lesion. The refinements of the problem are due to the secondary effects of vertebral rotation and the indirect deformities in the spinal column associated with paralysis and contractures in the limbs.

Nowadays we are more often concerned with the spinal deformity associated with myelomeningocele, and with the equally intransigent problem of idiopathic scoliosis. I believe there are many common factors in the development of these two conditions and in some cases of spinal deformity following cord injuryparticularly in the growing spine and in incomplete lesions, but the analogy should not be overstressed.

\section{FACTORS INFLUENCING SPINAL DEFORMITY}

Just as there is no single cause for idiopathic scoliosis, so also a number of factors must be considered in the development of spinal deformity consequent upon damage to the cord. It is convenient to list these as follows:

I. Degree of cord damage.

2. Level of cord lesion.

3. Age of the patient.

4. Effect of cord injury on the limbs.

5. Coincidental bony damage.

I. Degree of Cord Damage. When the cord injury is complete, the paralysis of the spinal musculature is symmetrical and in adults the tendency to develop spinal deformity is less than in the incomplete lesions. In children there is often a marked increase in the normal lumbar lordosis.

Incomplete lesions usually show asymmetrical paralysis which, when coupled with spasticity, may lead to one or a combination of the recognised spinal deformities, namely kyphosis, scoliosis, lordosis and rotational abnormalities. On theoretical grounds it would seem possible to predict which of these deformities will predominate. In clinical practice, however, this is not easy. The spasticity may be continuous or intermittent and it will vary in degree and response to treatment, so that its effect on the spinal column will be altered accordingly.

2. Level of Cord Lesion. In general terms the higher the lesion the greater is the potential for deformity. Usually the higher lesions exhibit a more widespread spasticity, even though the degree of spasticity may be less.

Sparing of the shoulder-girdle muscles and the serratus anterior in particular has an important effect on spinal alignment. Of even greater importance is the latissimus dorsi which by-passes any spinal lesion as high as $\mathrm{C}_{7}$ and acts as a link between the pelvis and the upper limbs (Guttmann, 1953). Paralysis of the abdominal muscles ( $\left.\mathrm{T}_{7}-\mathrm{I}_{2}\right)$ and of the quadratus lumborum ( $\mathrm{TI}_{2}-\mathrm{LI}_{1}$ ) predisposes to an increase in the lumbar lordosis, while spasticity, or perverted action of these 
muscles, may lead to kyphosis or a lumbar scoliosis. Abnormal action of the ilio-psoas muscle $\left(\mathrm{L}_{2}, 3,4\right)$ has a profound effect on the hip where it may produce a fixed flexion-adduction deformity, leading in severe cases to dislocation of the joint. In addition, contracture of the muscle may lead to a remarkable increase in the lumbar lordosis, and to pelvic obliquity when the contracture is asymmetrical or associated with dislocation of the hip.

Lesions below the conus do not commonly produce spinal deformity, though deformity may be associated with the local damage to the lumbar spine, or may result from neurologically produced contractures of the lower limbs.

A point to note about paralysis or spasticity of any particular muscle group is that the deforming effect is enhanced in the presence of abnormal action in other local muscle groups.

3. Age of the Patient. As already mentioned, deformities of the spine following cord injuries of comparable severity are much more common in children than in adults. In elderly patients residual spinal deformity is more often associated with co-incident bony damage, rather than with any neurological deficit.

With high lesions the development of a long ' $C$ ' curve is not unusual in children. Severe curves can seldom be adequately controlled by appliances, and may interfere with sitting in a wheelchair. Equally troublesome, but fortunately less common, is the 'collapsing spine' of tetraplegic children. In this condition the longitudinal muscles fail to regain tone, so that when such a child is sat up the spine collapses into an ' $S$ ' shaped curve.

4. Effect of Cord Injury on the Limbs. Paralysis of the upper limbs is seldom a cause of fixed deformity in the spine. Alteration in posture of the lower limbs, however, may have a profound effect on the vertebral column. Deformities of the joints of the lower limbs brought about by spasm, contractures, septic arthritis of hip or knee, or myositis ossificans are still encountered in a minority of patients, in whom they may produce secondary spinal deformity not due to the paralysis alone.

Flexion contracture of the hips increases the forward inclination of the pelvis and, more importantly, increases the compensatory lumbar lordosis. Unequal contracture of the hips almost invariably leads to a scoliosis.

Flexion contracture of the knee may lead to a secondary contracture of the hip and the train of events already considered.

$A$ contracted ilio-tibial band is encountered, albeit rarely, as a deforming force in patients who have suffered a cord injury. A taut ilio-tibial band, perceived by deep palpation while adducting and attempting to extend the thigh, is a potent source of lower limb deformity. Because the band lies in a plane anterior and lateral to the hip, a flexion abduction contracture quickly develops. This in turn leads to pelvic obliquity and a lumbar scoliosis.

Pelvic obliquity may be one of the most troublesome deformities following on injury to the spinal cord. If an abduction contracture of the hip develops while the patient is recumbent, the pelvis will develop an oblique disposition in relation to the vertical axis of the spine. When the patient is placed erect, semi-erect or sitting, the pelvis drops on the contracted side and rises on the opposite side. With the passage of time the muscles on the affected side become overstretched, and those on the opposite side become shortened. If this state of affairs is not 
corrected, skeletal changes will fix the deformity and will lead to a scoliosis convex towards the side of the affected (more paralysed) hip. A similar state of affairs may develop with intractable pressure sores around the hip, in septic arthritis, and with extensive para-articular ossifications.

5. Coincidental Bony Damage. In the cervical spine the loss of alignment due to an unreduced subluxation or dislocation, the loss of lordosis due to collapse

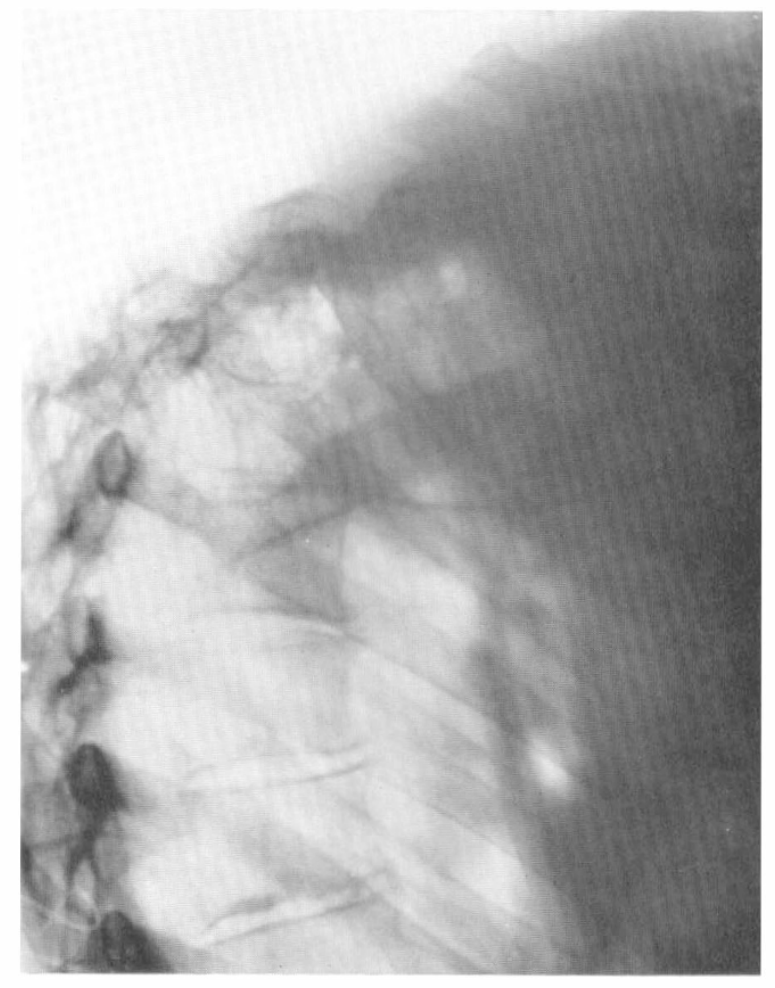

FIG. 3

Severe compression fracture D.V. 4 in 67-year-old female two years after injury. Note degree of collapse.

of a vertebral body, or due to progressive deformity, is seldom of consequence when regarded merely as a deformity. The loss of alignment may have other neurological implications, which will be discussed in a later section.

Fractures of the dorsal spine heal with some vertebral body collapse, so that in severe injuries some kyphosis is inevitable. This is especially true in the elderly whose bones are porotic and in whom the period of recumbency needs to be kept as short as possible (figs 3 and 4 ).

Injuries of the lumbar spine are usually associated with some degree of kyphosis or scoliosis. The deformity may be lessened by postural reduction, but in my experience severe compression fractures and fracture-dislocations consolidate 
only in a position of deformity. Internal fixation offers no guarantee that the position will be maintained.

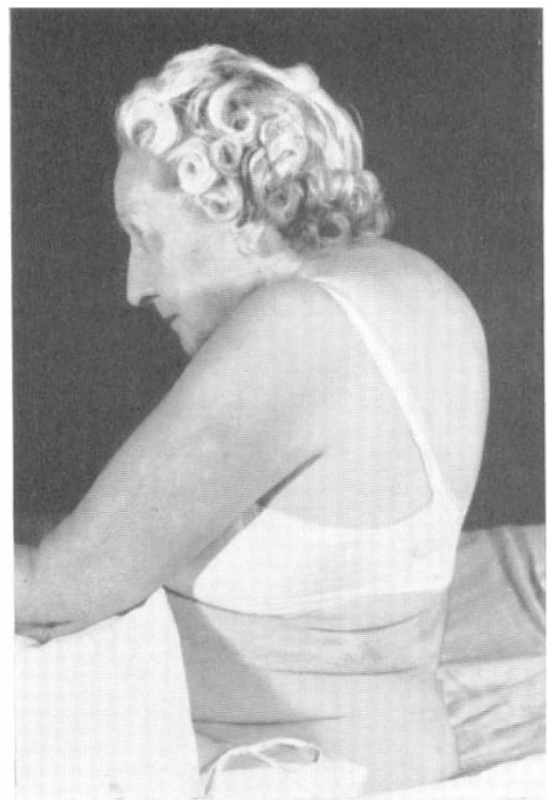

FIG. 4

Clinical appearance of patient whose $\mathrm{X}$-ray is shown in Figure 3 .

\section{TREATMENT}

Most of the skeletal problems following injury to the spinal cord are preventable, and many of the problems discussed in this paper need not occur. I do not propose to discuss the initial management of the injured patient and the allimportant role of the physiotherapist and remedial gymnast, except in a general way. The treatment of spasticity is a subject in itself, beyond the scope of the present paper.

It is hardly necessary to point out that severe degrees of spinal deformity are compatible with an active tetraplegic or paraplegic life, whether the patient is confined to a wheelchair or ambulant on crutches. It is often salutary to examine a series of X-ray films taken for routine pyelography and to correlate the scoliosis, or other spinal deformity, so often present with the otherwise fully rehabilitated patient.

With a really active rehabilitation programme it is remarkable how a physiotherapist can overcome apparently incorrigible deformities by resistance exercises and hydrotherapy. Nevertheless, there are some patients where the deformity is so fixed that little response to physiotherapy can be expected. Only when all the non-operative measures have been exploited should surgical intervention be 
considered. At the best of times this is hazardous in such patients. The individual surgical procedures are not random selections and are decided upon only when the patient as a whole, and all aspects of his problem, have been examined under the widest terms of reference.

Flexion contracture of the hips responds to division of the anterior capsule and soft tissues over the front of the hip, combined with division of the ilio-psoas muscle. More severe cases due to a septic arthritis may require excision of the upper part of the femur to combat the infection. Patients presenting with widespread para-articular ossification are often improved by radical excision of bony plaques at the appropriate time.

Severe flexion contracture of the knee may be treated by posterior capsulotomy and radical division of soft tissues, with due respect for, and sparing only, the neurovascular structures.

A contracted ilio-tibial band cannot be adequately corrected except by surgical means. A large section of the band (tract), including its intermuscular septum and the tensor fasciae femoris muscle, should be excised. This should be combined with an extensive soft tissue release around the hip and knee.

In neglected cases pelvic obliquity demands, firstly, correction of the abducted hip by soft tissue release and secondly, corrective abduction osteotomy of the opposite (adducted) hip, preferably with internal fixation.

If fixed lumbar scoliosis remains a problem a transabdominal extraperitoneal wedge resection of a vertebral body, with fixation by a heavy plate of the Liverpool pattern, probably offers the best solution (Roaf, I968). However, I have not yet met a patient in whom the lumbar deformity, following a spinal cord injury, was severe enough to require so drastic a measure. Roaf has been obliged to adopt this procedure in a number of patients who have recovered from very severe intracranial injuries and infections.

Severe kypho-scoliosis or lordosis due to paralysis of spinal or abdominal muscles, and not associated with uncorrected contributory deformities, may require correction and spinal fusion. For these exceptional cases procedures applicable to scoliosis from other causes apply. It is important to note that plaster jackets or rigid braces are contra-indicated, so that some form of internal fixation is necessary. The Harrington rod is a suitable device for long curves, and the Liverpool plate for shorter and more acute curves. Such a course may be dictated (especially in tetraplegic children) by respiratory embarrassment, inability to wear a corset, or to sit in comfort in a wheelchair. It goes without saying that surgery in such cases is a last resort.

Bony deformity associated with painful mal-alignment of the spine may require late fusion, a state of affairs usually seen only if the spinal cord injury is incomplete. As a general rule correction of the deformity should not be undertaken, as there is always the possibility of adversely affecting the neurological picture. In exceptional cases gross unreduced bony deformity may require minor procedures, such as excision of bony prominences to prevent the development of pressure sores. The degree of stability and the absence of pain in grossly unreduced fracture-dislocations some months after the injury is often remarkable (figs 5, 6 and 7).

Late deformity in the cervical region presents a special problem. A number of important studies have appeared on this controversial subject in recent years (Beatson, I963; Braakman \& Vinken, I968). 


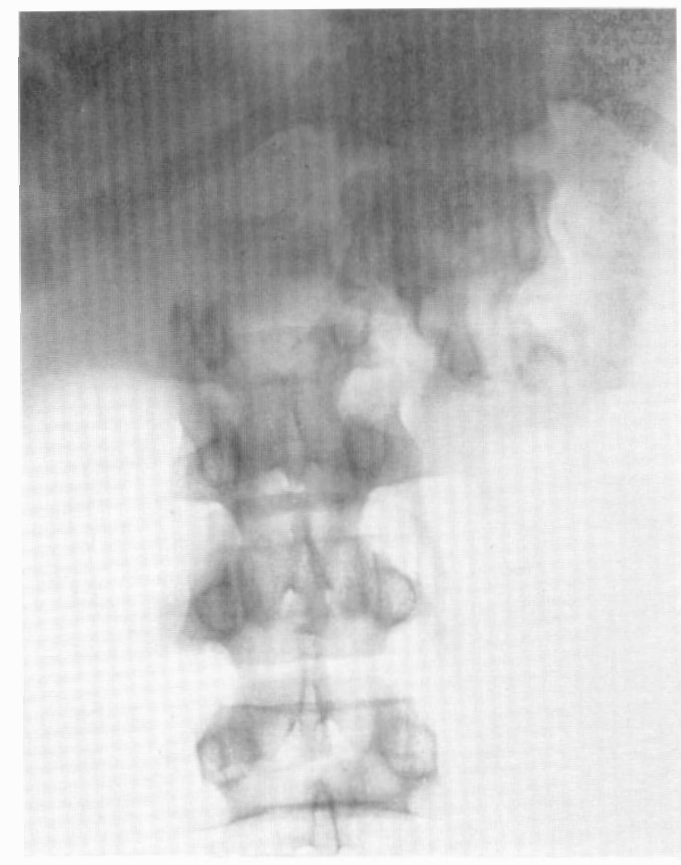

FIG. 5

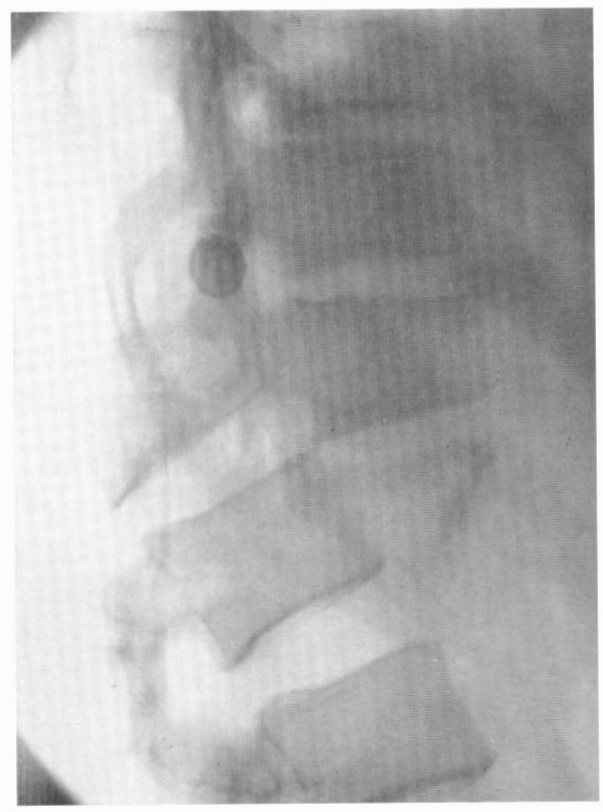

FIG. 6

Figs. 5 and 6.-Grotesque fracture-dislocation dorso-lumbar junction with complete paraplegia. Stable painless spine six months after injury.

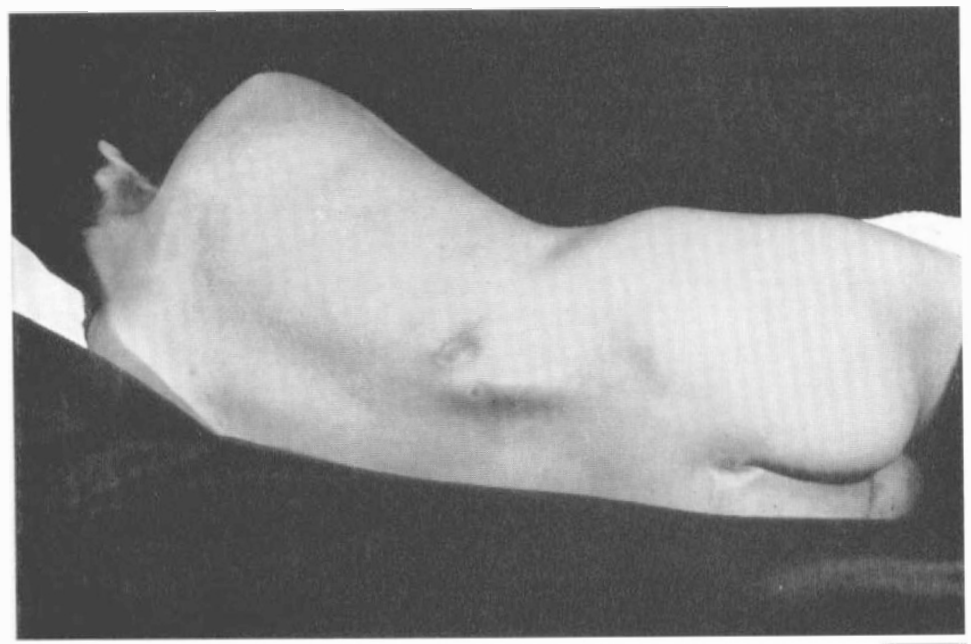

FIG. 7

Same patient as in Figures 5 and 6 before excision of bony prominences. 
In the author's view the majority of these neck injuries are stable after four months, but a surgeon may decide to play for safety and therefore advise the patient to wear a well-fitting collar for a further few months. Three clinical, as distinct from radiological, features may dictate surgical intervention. These are: severe pain in the neck, persisting symptoms of root compression, and progressive cord signs. In such cases cervical fusion, without attempting to correct the deformity, should be considered in conjunction with the need for foraminotomy to relieve root compression, or laminectomy for progressive cord compression.

Indiscriminate fusion of the cervical spine in the acute phase is condemned. Apart from open wounds or progression of an initially incomplete lesion, both of which demand exploration, it is difficult to see the need for immediate surgical intervention, much less the indication for immediate fusion.

Patients in whom the cord lesion is manifestly complete immediately after the injury, and remains so for 48 hours, should not be subjected to surgery. Even in the most skilled hands operative fusion increases the respiratory hazards and the liability to paralytic ileus.

Faced with the catastrophic situation of a complete cord lesion at the cervical level it is understandable that all concerned are anxious that everything possible should be done to reverse the tragic state of affairs. In the light of our present knowledge it is difficult to see how fusing the spine can contribute to the patient's well-being.

In this context it would be of the utmost value to find a method of repair for the severely damaged spinal cord but, as I see it, much more animal experimental work is necessary to justify a direct surgical approach.

A greater understanding of the skeletal effects of cord injury and the management of their sequelae adds to the comfort and usefulness of these severely handicapped patients.

\section{ACKNOWLEDGEMENT}

I am greatly indebted to Professor Robert Roaf and Mr. D. Ll. Griffiths for their interest and helpful criticism in the preparation of this paper. I also wish to thank Mr. Beverley Southern for the illustrations.

\section{REFERENCES}

Beatson, T. R. (1963). F. Bone ft. Surg. 45-B, 2 I.

BRAakman, R. \& Vinken, P. J. (1968). F. Bone ft. Surg. 50-B, 52.

GutTManN, L. (1952). F. nerv. ment. Dis. I 1 6, 957 (1952).

Guttmann, L. (1953). History of the Second World War. Surgery. Edited by Sir Z. Cope. H.M. Stationery Office I 21, I 52 .

Roaf, R. (I966). Scoliosis. Edinburgh: E. \& S. Livingstone.

RoAf, R. (I968). Personal Communication. 